\title{
Mechanistic insights into chemical and photochemical transformations of bismuth vanadate photoanodes
}

Francesca M. Toma ${ }^{1,2}$, Jason K. Cooper ${ }^{1,2}$, Viktoria Kunzelmann ${ }^{1,3}$, Matthew T. McDowell ${ }^{4,5, \dagger}$, Jie Yu $\mathbf{u}^{1,2}$, David M. Larson ${ }^{1,2}$, Nicholas J. Borys ${ }^{6,7}$, Christine Abelyan ${ }^{1,2}$, Jeffrey W. Beeman ${ }^{1,7}$, Kin Man Yu ${ }^{7, \dagger}$, Jinhui Yang ${ }^{1,7}$, Le Chen ${ }^{1,7}$, Matthew R. Shaner ${ }^{4,5}$, Joshua Spurgeon ${ }^{4,5, \dagger}$, Frances A. Houle ${ }^{1,2}$, Kristin A. Persson ${ }^{1,8,9}$ \& Ian D. Sharp ${ }^{1,2}$

Artificial photosynthesis relies on the availability of semiconductors that are chemically stable and can efficiently capture solar energy. Although metal oxide semiconductors have been investigated for their promise to resist oxidative attack, materials in this class can suffer from chemical and photochemical instability. Here we present a methodology for evaluating corrosion mechanisms and apply it to bismuth vanadate, a state-of-the-art photoanode. Analysis of changing morphology and composition under solar water splitting conditions reveals chemical instabilities that are not predicted from thermodynamic considerations of stable solid oxide phases, as represented by the Pourbaix diagram for the system. Computational modelling indicates that photoexcited charge carriers accumulated at the surface destabilize the lattice, and that self-passivation by formation of a chemically stable surface phase is kinetically hindered. Although chemical stability of metal oxides cannot be assumed, insight into corrosion mechanisms aids development of protection strategies and discovery of semiconductors with improved stability.

\footnotetext{
${ }^{1}$ Joint Center for Artificial Photosynthesis, Lawrence Berkeley National Laboratory, 1 Cyclotron Road, Berkeley, California 94720, USA. ${ }^{2}$ Chemical Sciences Division, Lawrence Berkeley National Laboratory, 1 Cyclotron Road, Berkeley, California 94720, USA. ${ }^{3}$ Walter Schottky Institut and Physik Department, Technische Universität München, Am Coulombwall 4, 85748 Garching, Germany. ${ }^{4}$ Joint Center for Artificial Photosynthesis, California Institute of Technology, 1200 East California Boulevard, Pasadena, California 91125, USA. ${ }^{5}$ Division of Chemistry and Chemical Engineering, California Institute of Technology, 1200 East California Boulevard, Pasadena, California 91125, USA. ${ }^{6}$ Molecular Foundry, Lawrence Berkeley National Laboratory, 1 Cyclotron Road, Berkeley, California 94720, USA. ${ }^{7}$ Materials Sciences Division, Lawrence Berkeley National Laboratory, 1 Cyclotron Road, Berkeley, California 94720, USA. ${ }^{8}$ Energy Technologies Area, Lawrence Berkeley National Laboratory, 1 Cyclotron Road, Berkeley, California 94720, USA. ${ }^{9}$ Materials Science and Engineering, University of California, Berkeley, 210 Hearst Memorial Mining Building, Berkeley, California 94720, USA. † Present addresses: G.W. Woodruff School of Mechanical Engineering and School of Materials Science and Engineering, Georgia Institute of Technology, Atlanta, Georgia 30332, USA (M.T.M.); Department of Physics and Materials Science, City University of Hong Kong, Kowloon, Hong Kong (K.M.Y.); Conn Center for Renewable Energy Research, University of Louisville, Louisville, Kentucky 40292, USA (J.S.). Correspondence and requests for materials should be addressed to F.M.T. (email: fmtoma@lbl.gov) or to I.D.S. (email: idsharp@lbl.gov).
} 
$\mathrm{P}$ hotoelectrochemical conversion of solar to chemical energy is an attractive approach for sustainable production of hydrogen and hydrocarbon fuels ${ }^{1,2}$. However, harnessing light to split water involves thermodynamic and kinetic challenges, with major efficiency losses associated with oxygen production $^{1-3}$. Furthermore, material stability is often poor under the harsh conditions in which this reaction is performed. Therefore, much attention has been devoted to the development of $n$-type thin film semiconductors that are chemically stable, can efficiently harvest sunlight and can oxidize water when coupled to appropriate catalysts ${ }^{3}$. Among available candidates for photoanodes, metal oxides are a promising class of potentially durable materials, as they are usually less readily oxidized than water ${ }^{3,4}$. However, many of the most stable metal oxide semiconductors possess wide bandgaps that render them impractical for solar energy conversion. Thus, a central challenge is the discovery and development of new semiconductors that are stable and have bandgaps suitable for efficiently capturing solar energy. In recent times, the quest for such materials has been accelerated by advances in highthroughput computation for prediction of electronic structure and thermodynamic stability of semiconductors ${ }^{4}$. Understanding the present limits of such computational approaches and improving their ability to predict the complex behaviours of real materials requires direct comparison with experiments using known semiconductors.

Although thermodynamic considerations provide a starting point for evaluating (photo)electrochemical stability of materials under the conditions present in solar fuel generators, kinetic factors can also play a dominant role on achievable operational durability. Indeed, some materials exhibit chemical instabilities that cannot be explained by thermodynamic considerations alone. One such material is $\mathrm{BiVO}_{4}$, which has been actively investigated as one of the highest performance oxide photoanodes for water splitting $^{5}$. This material has garnered significant interest, because it absorbs in the visible range, with a bandgap of $2.5 \mathrm{eV}$ (ref. 6), and has conduction and valence band positions- $\sim 4.77$ and $7.27 \mathrm{eV}$ below the vacuum level, respectively-that are well-suited for generating large photovoltages and driving the oxygenevolution reaction (OER) ${ }^{7}$. The calculated oxidation potential of $\mathrm{BiVO}_{4}$ has been predicted to be slightly more positive $(1.24 \mathrm{~V}$ versus reversible hydrogen electrode, $\mathrm{RHE}$ ) than the water oxidation potential $\left(\mathrm{O}_{2} / \mathrm{H}_{2} \mathrm{O}, 1.23 \mathrm{~V}\right.$ versus $\left.\mathrm{RHE}\right)$, suggesting that this material could be resistant to photoinduced corrosion under water splitting conditions ${ }^{4}$. In contrast to this prediction, this material suffers from photoelectrochemical instability in both near-neutral and alkaline $\mathrm{pH}$ ranges ${ }^{8-10}$. Although photocorrosion of $\mathrm{BiVO}_{4}$ has been correlated with vanadium leaching from the surface into the electrolyte solution ${ }^{9,11,12}$, the mechanisms of photodegradation and the associated chemical transformations have not yet been identified. This poor stability undermines its practical use in large-scale integrated devices ${ }^{8}$.

In this work, we establish a methodology for systematically assessing the chemical and photochemical stabilities of semiconductors and apply it to a visible-light-absorbing semiconductor, $\mathrm{BiVO}_{4}$. We investigate chemical and photochemical corrosion pathways using $\mathrm{BiVO}_{4}$ thin films at near-neutral and at high $\mathrm{pH}$, thereby simulating possible operating conditions in solar fuel devices ${ }^{13}$. These measurements demonstrate that $\mathrm{BiVO}_{4}$ is susceptible to chemical corrosion that is accelerated by illumination (photodegradation). Electron microscopy indicates that photodegradation initiates at and propagates from surfaces and grain boundaries, resulting in extensive dissolution of the films. Given the existence of thermodynamically stable bismuth oxides predicted by Pourbaix diagrams under these conditions, formation of a chemically stable surface layer was expected but not observed. Therefore, bulk dissolution of the film cannot be described by thermodynamic arguments. This finding is confirmed by in situ electrochemical atomic force microscopy (EC-AFM), which reveals that degradation under operating conditions occurs via dissolution of the film, starting at exposed facets of grains in polycrystalline thin films. To understand this chemical behaviour, we use computational methods to examine both the illuminated and dark stability. This analysis indicates that accumulation of photogenerated holes at the surface leads to structural destabilization and chemical attack via vanadium loss into solution. Although self-passivation of the material with bismuth oxide would be expected, thermodynamically stable phases of this oxide require significant structural transformations from the low-density $\mathrm{V}$-leached state and are not accessible at room temperature. Therefore, photocorrosion results in bulk dissolution of the material. Our measurements reveal that nonequilibrium and kinetic factors dominate chemical and photochemical degradation processes for this commonly studied semiconductor material, and these factors must be considered for prediction of new materials that possess desired combinations of stability and efficiency. Furthermore, the kinetic accessibility of self-passivating surface layers, which broaden the range of available corrosion protection strategies, must be considered for engineering the interfaces of materials possessing lower intrinsic stability. These findings can be used to guide extensions of theory to consider intermediate (photo)chemical states and may provide enhanced predictive power for evaluating and interpreting the stabilities of promising new semiconductors for solar fuel applications. In addition, improved understanding of mechanisms governing photochemical stability will guide development of experimental strategies for stabilizing chemically sensitive semiconductors.

\section{Results}

Evaluation of photoelectrochemical stability of bismuth vanadate. Evaluating the stability of materials for photoelectrochemical water splitting requires testing under conditions that are relevant to practical devices. Although near-neutral conditions are often used for testing individual water splitting photoelectrodes and place less severe constraints on material stability relative to acidic and alkaline conditions, current integrated solar water splitting devices require either electrolyte recirculation or more extreme $\mathrm{pH}$ environments to eliminate severe $\mathrm{pH}$ gradients and ohmic losses ${ }^{14}$. Much work has been reported on the photoelectrochemical properties of $\mathrm{BiVO}_{4}$ at near neutral $\mathrm{pH}$, where stabilization has been realized via integration of OER catalysts, such as cobalt phosphate $(\mathrm{CoPi})^{15}$ and iron oxyhydroxide $(\mathrm{FeO}(\mathrm{OH}))^{8,16,17}$. However, strongly alkaline conditions are often desirable for avoiding hydroxide ion depletion near the electrode and high overpotentials due to ohmic losses in water splitting devices that separate chemical products $^{14,18}$. Fewer reports have addressed the characteristics of $\mathrm{BiVO}_{4}$ under alkaline conditions ${ }^{9,10}$. Notably, a dual layer $\mathrm{TiO}_{2} / \mathrm{Ni}$ coating was recently demonstrated to significantly impede degradation at high $\mathrm{pH}^{9}$. Under all testing conditions, the long-term stability of this material remains a question and understanding of the factors governing corrosion and photocorrosion may provide a means of predicting lifetime, preventing failure and improving strategies for catalyst integration. Importantly, understanding chemical and photochemical interactions of state-of-the-art materials, such as $\mathrm{BiVO}_{4}$, provides a basis for rational design of new materials by identifying kinetic degradation pathways that can be evaluated computationally.

Because of its different $p$ Ka values, phosphate buffer allows for the study of stability at various $\mathrm{pH}$ conditions that are relevant 
for water-splitting applications, while maintaining equal ionic strength. Therefore, we chose to study the stability of $\mathrm{BiVO}_{4}$ using $1 \mathrm{M}$ potassium phosphate $(\mathrm{KPi})$ buffer at $\mathrm{pH} 6.8$ and at $\mathrm{pH}$ 12.3 under biased and unbiased dark and illuminated (AM 1.5, $100 \mathrm{~mW} \mathrm{~cm}^{-2}$ ) conditions.

For this study, we selected undoped $\mathrm{BiVO}_{4}$ deposited by the spin coating method, because it allows for reproducible fabrication of large area planar thin films. As such, spin-coated $\mathrm{BiVO}_{4}$ is well suited for achieving reliable statistics, drawing comparisons between bulk and surface effects, and analysing the impact of degradation on surface chemistry and morphology. However, to confirm that the findings presented here are generally valid for the material synthesized via other methods, a variety of undoped and doped thin films deposited by different techniques, namely electrodeposition $^{16}$, reactive sputtering ${ }^{17}$ and spray pyrolysis ${ }^{19}$, were also tested under similar conditions and yielded qualitatively similar stability results (Supplementary Figs 1 and 2).

Deposition by spin coating of molecular organic precursors onto fluorine-doped tin oxide (FTO) layers yields purephase monoclinic scheelite $\mathrm{BiVO}_{4}$ (Supplementary Fig. 3). Scanning electron microscopy (SEM) shows that the films are characterized by average grain sizes of $153 \pm 25 \mathrm{~nm}$ (Supplementary Fig. 3). Rutherford backscattering spectrometry (RBS) indicates a film thickness of $\sim 51.6 \pm 3.2 \mathrm{~nm}$ and is in excellent agreement with cross-sectional transmission electron microscopy (TEM) analysis, which reveals a thickness of $\sim 54 \mathrm{~nm}$ (Supplementary Fig. 3). In addition to thickness, RBS was used to evaluate thin film composition and revealed a stoichiometric ratio of $1: 1 \mathrm{Bi}: \mathrm{V}$. Importantly, complementary X-ray photoelectron spectroscopy (XPS) measurements indicate significant differences in surface stoichiometry compared with bulk, with a surface atomic ratio of $1.27: 1$ of $\mathrm{Bi}: \mathrm{V}$ and an overall composition of 21.6 at.\% $\mathrm{Bi}, 17.0$ at.\% $\mathrm{V}$ and 61.3 at.\% $\mathrm{O}$ (compared with the ideal stoichiometric composition of 16.7 at.\% $\mathrm{Bi}, 16.7$ at.\% $\mathrm{V}$ and 66.6 at.\% O, respectively; Supplementary Table 1).

As a starting point for the corrosion studies, the performance of $\mathrm{BiVO}_{4}$ thin films was evaluated at different $\mathrm{pH}$ values in the presence of $0.1 \mathrm{M}$ sodium sulfite as a hole scavenger. As sodium sulfite oxidation is thermodynamically favoured and is kinetically facile with respect to water oxidation, this approach allows for determination of the charge separation and extraction efficiency in $\mathrm{BiVO}_{4}$, without influence by the intrinsically poor catalytic activity of its surface ${ }^{8}$. Figure la show a comparison of photocurrent density versus potential $(J-E)$ anodic sweeps of pristine $\mathrm{BiVO}_{4}$ obtained at $\mathrm{pH} 6.8$ and at $\mathrm{pH} 12.3$, respectively. The sample measured at $\mathrm{pH} 6.8$ before its degradation is characterized by an open circuit potential of $0.35 \mathrm{~V}$ versus RHE and a current density of $2.2 \mathrm{~mA} \mathrm{~cm}^{-2}$ at $1.23 \mathrm{~V}$ versus RHE. The sample measured at $\mathrm{pH} 12.3$ before stability testing shows an identical open circuit potential, but exhibits a significantly reduced fill factor and a smaller photocurrent of $1.8 \mathrm{~mA} \mathrm{~cm}^{-2}$ at $1.23 \mathrm{~V}$ versus RHE.

The reduced fill factor and photocurrent density observed under alkaline testing conditions suggest that rapid chemical modification of $\mathrm{BiVO}_{4}$ at high $\mathrm{pH}$ (see below) introduces additional recombination centres at the surface and/or an insulating surface layer that increases the resistance to charge transfer across the interface ${ }^{3,20}$. Furthermore, analysis of cyclic voltammograms (CVs) reveals an increase of the $J-E$ hysteresis (see Supplementary Fig. 4) at high $\mathrm{pH}$. This hysteresis is consistent with surface state charging and may also be affected by modified interfacial energetics arising from changing occupancies of surface states during illuminated cycling. The pronounced hysteresis under alkaline conditions further suggests that chemical modification at high $\mathrm{pH}$ introduces electronically active surface states.
Chronoamperometry measurements show the change of photocurrent from $\mathrm{BiVO}_{4}$ thin films as a function of time at fixed applied potential of $1.23 \mathrm{~V}$ versus RHE at both pH 6.8 and 12.3 in the absence of hole scavenger (Fig. 1b). In both cases, these data are characterized by a rapid decrease of the photocurrent during the first ten seconds (inset). This effect is significantly accelerated under alkaline conditions and is consistent with both the reduced fill factor and increased hysteresis relative to near neutral conditions, as described above. The initial fast photocurrent decay is followed by a slower decay over the course of the $60 \mathrm{~min}(\mathrm{pH}$ 6.8) and $20 \mathrm{~min}(\mathrm{pH} 12.8)$ measurements.

After the $1 \mathrm{~h}$ chronoamperometry test in phosphate buffer at near neutral $\mathrm{pH}$, cyclic voltammetry of degraded $\mathrm{BiVO}_{4}$ was again performed in the presence of sacrificial hole acceptor (Fig. 1c). We observe an anodic shift in the photocurrent onset potential of about $0.1 \mathrm{~V}$, as well as decreases of both the current density and fill factor. Furthermore, analysis of CVs reveals that significant hysteresis is present following sustained operation (Supplementary Fig. 4). This finding suggests that consumption of the films by photocorrosion is also accompanied by the introduction of higher capacitance, probably due to charging of surface defect states, even at moderate $\mathrm{pH}$.

Similarly, the 20 min chronoamperometric stability test at high $\mathrm{pH}$ reduces the photoelectrochemical performance of this material, with an anodic shift of the onset potential $(\sim 0.1 \mathrm{~V})$ and decrease of the current density. Hysteresis remains approximately constant, supporting the finding that chemical transformation of the surface occurs immediately on immersion in the alkaline electrolyte. Interestingly, although the photocurrents were observed to significantly decline after chronoamperometric testing at $1.23 \mathrm{~V}$ versus $\mathrm{RHE}$ in the absence of sacrificial reagent (by $\sim 75 \%$ at $\mathrm{pH} 6.8$ and $\sim 95 \%$ at $\mathrm{pH} 12.3$, Supplementary Fig. 5), subsequent $J-E$ characterization in the presence of $\mathrm{Na}_{2} \mathrm{SO}_{3}$ revealed much less pronounced reductions in the photocurrent density (Fig. 1). As neither the local nor bulk $\mathrm{pH}$ changes can account for the observed behaviour (Supplementary Note 1), this observation suggests that, in addition to introducing electronic defect states, chemical modification of the surface also reduces the native catalytic activity of the surfaces for water oxidation.

As described below, elemental analysis suggests that photocorrosion may result in enrichment of bismuth oxide on the surface. As oxides of bismuth are known to exhibit poor catalytic activity for water oxidation ${ }^{21}$, chronoamperometric testing in pure phosphate buffer yields dramatic performance degradation under sustained operation. However, it is important to note that these measurements convolve catalytic activity with light capture and charge extraction efficiencies. Therefore, comparison of $J-E$ curves obtained with a sacrificial hole acceptor, such as those shown in Fig. 1, more accurately represent the effects of photocorrosion and chemical modification on semiconductor performance for solar energy conversion.

Compositional analysis of bismuth vanadate photoanodes. To obtain insights into the mechanism of photodegradation and understand the changes in photoelectrochemical characteristics under operational conditions, we performed a compositional analysis of thin films before and after the chronoamperometric testing. As described above, for the case of pristine $\mathrm{BiVO}_{4}$ thin films, a combination of XPS and RBS was used to analyse differences between the surface and bulk composition, which can be affected by surface chemistry and have an important impact on interfacial charge transport, as well as catalysis.

As summarized in Table 1, RBS indicates that the bulk ratio of $\mathrm{Bi}: \mathrm{V}$ is stoichiometric, with a ratio of $1: 1$, and does not change 

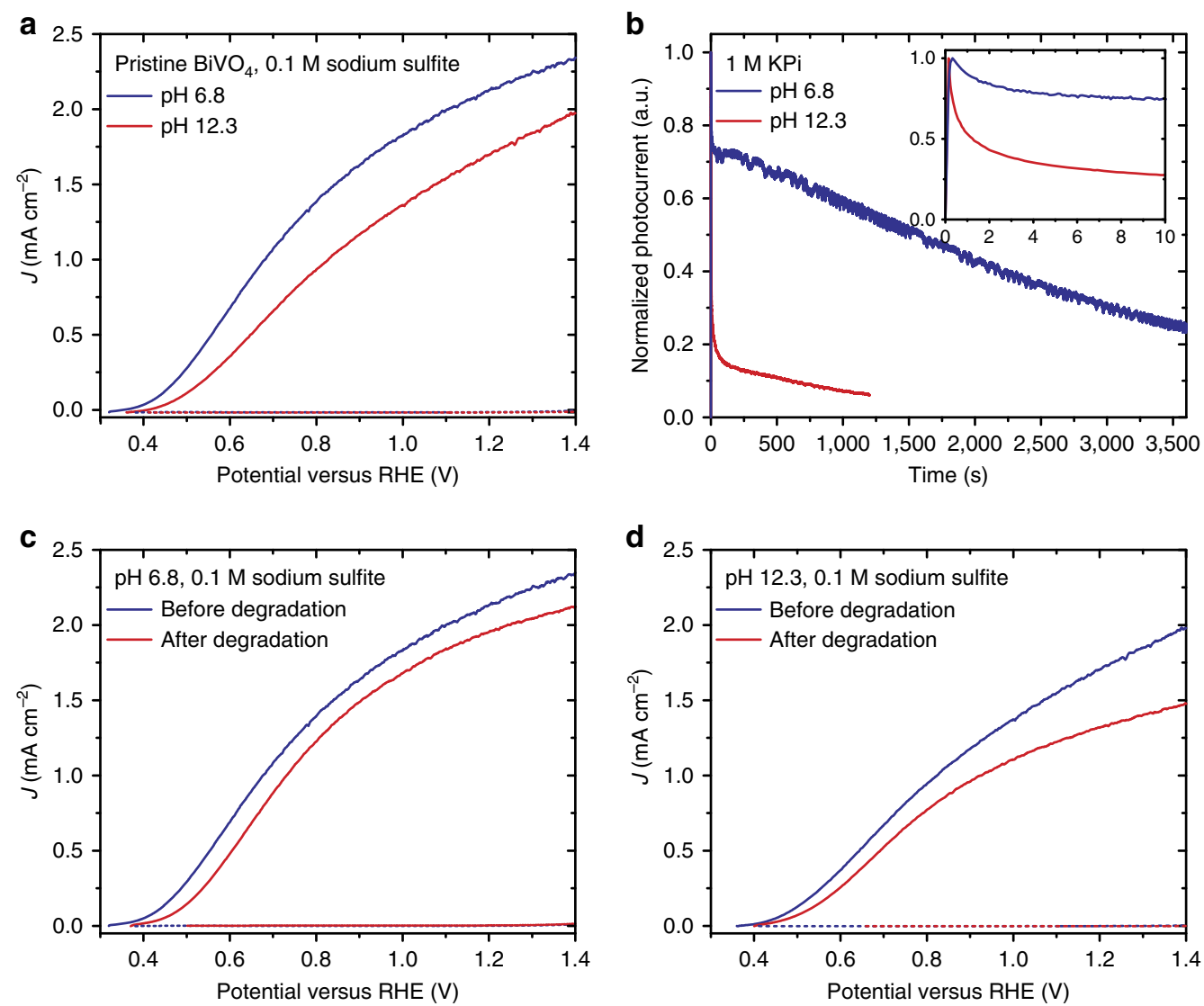

Figure 1 | Photoelectrochemical characterization of $\mathbf{B i V O}_{\mathbf{4}}$ electrodes in $\mathbf{1} \mathbf{M} \mathbf{~ K P i}$ buffer. (a) Photoelectrochemical J-E characterization of pristine $\mathrm{BiVO}_{4}$ thin films in solution containing $0.1 \mathrm{M}$ sulfite at $\mathrm{pH} 6.8$ (blue curve) and $\mathrm{pH} 12.3$ (red curve) under light (solid curve) and dark (dotted curve). (b) Chronoamperometry of pristine $\mathrm{BiVO}_{4}$ thin films in pure $\mathrm{KPi}$ solution (no sulfite present) at $\mathrm{pH} 6.8$ (blue curve) and pH 12.3 (red curve) at a fixed applied bias of $1.23 \mathrm{~V}$ versus RHE. (c) $\mathrm{J}$-E characteristics of $\mathrm{BiVO}_{4}$ thin films in solution containing $0.1 \mathrm{M}$ sulfite at pH 6.8 under light (solid curve) and dark (dotted curve) before (blue curve) and after (red curve) testing. (d) J-E characteristics of $\mathrm{BiVO}_{4}$ thin films in solution containing $0.1 \mathrm{M}$ sulfite at pH 12.3 under light (solid curve) and dark (dotted curve) before (blue curve) and after (red curve) testing. All measurements under illumination were performed using simulated AM 1.5 light (Solar Light) adjusted to $100 \mathrm{~mW} \mathrm{~cm}^{-2}$ using a calibrated Si photovoltaic cell (SolarSim calibration, Newport).

Table 1 | Bulk and surface composition analysis.

\begin{tabular}{llccc} 
& Testing condition & Bi (at.\%) & V (at.\%) & Thickness $^{\star}$ (nm) \\
\hline Ideal $^{\dagger}$ & & $16.7(1)$ & $16.7(1)$ & \\
RBS & Pristine & $14.3 \pm 0.5$ & $14.5 \pm 1.3$ & $51.6 \pm 3.2$ \\
& pH 6.8 & $14.5 \pm 0.7$ & $14.1 \pm 0.7$ & $44.1 \pm 3.7$ \\
& pH 12.3 & $11.1 \pm 1.5$ & $11.1 \pm 1.5$ & $37.4 \pm 1.8$ \\
XPS & Pristine & $23.5 \pm 1.5$ & $16.3 \pm 0.7$ & \\
& pH 6.8 & $25.3 \pm 0.7$ & $15.4 \pm 0.4$ & \\
& pH 12.3 & $26.7 \pm 1.6$ & $14.0 \pm 0.5$ & \\
\end{tabular}

RBS, rutherford backscattering spectrometry; XPS, X-ray photoelectron spectroscopy. *Thickness calculated from the number of atoms detected by RBS, where bulk density of $\mathrm{BiVO}_{4}$ is assumed 48 . Tests were performed for $60 \mathrm{~min}$ at $\mathrm{pH} 6.8$ and $20 \mathrm{~min}$ at $\mathrm{pH} 12.3$. $\dagger$ ldeal atomic ratio and ideal stoichiometric ratio in parenthesis. Composition analysis including $\mathrm{O}$ at.\% is reported in Supplementary Table 1.

during the degradation process. From fitting of RBS data, we calculated the thickness of the pristine $\mathrm{BiVO}_{4}$ layer $(51.6 \pm 3.2 \mathrm{~nm})$, as well as of the degraded samples $(44.1 \pm 3.7 \mathrm{~nm}$ at $\mathrm{pH} 6.8$ and $37.4 \pm 1.8 \mathrm{~nm}$ at $\mathrm{pH} 12.3)$. The progressive thinning of the layer during sustained operation under illumination indicates photocorrosion of the films over time, with nominal rates of $0.125 \mathrm{nmmin}^{-1}$ at $\mathrm{pH} 6.8$ and $0.71 \mathrm{nmmin}^{-1}$ at $\mathrm{pH} 12.3\left(7.5 \mathrm{nmh}^{-1}\right.$ at $\mathrm{pH} \quad 6.8$ and $42.6 \mathrm{~nm} \mathrm{~h}^{-1}$ at $\mathrm{pH}$ 12.3). Thus, photocorrosion is accelerated under alkaline conditions. However, it must be noted that the
RBS model assumes a homogenous corrosion of the film, which is not supported by the SEM and TEM results (vide infra). In addition, quantification of bulk oxygen content by RBS in the degraded samples was complicated by the underlying FTO.

In contrast to the constant bulk stoichiometry determined by RBS, important changes of the surface composition are observed by XPS (Fig. 2). Comparison of photoemission spectra obtained before and after photoelectrochemical stability testing reveals no significant changes in the $\mathrm{Bi} 4 f$ or the $\mathrm{V} 2 p$ core level lineshapes. Analysis of the $\mathrm{O} 1 s$ spectral region reveals a main component at $529.4 \pm 0.3 \mathrm{eV}$ from oxygen in the thin film. However, a shoulder at $531.7 \pm 0.1 \mathrm{eV}$ emerges following illuminated chronoamperometric testing. As discussed below, SEM and TEM analyses prove that the films become discontinuous after stability testing. Therefore, photoelectrons from regions of bare FTO contribute to the overall spectrum. Comparison with a reference FTO standard confirms this and enables assignment of the $531.7 \pm 0.1 \mathrm{eV}$ component to oxygen in the FTO substrate. As summarized in Table 1, analysis of XPS core-level intensity changes shows that surfaces become enriched with $\mathrm{Bi}$ and slightly depleted of $\mathrm{V}$ following chronoamperometric testing at both $\mathrm{pH}$ 6.8 and 12.3. Loss of $\mathrm{V}$ from the surface is consistent with prior reports of $\mathrm{BiVO}_{4}$ composition modification under aqueous conditions $^{9,11}$. However, the associated enrichment of $\mathrm{Bi}$, on an atomic per cent basis, suggests the possible formation of a terminal surface of bismuth oxide, such as $\mathrm{Bi}_{2} \mathrm{O}_{3}$ or $\mathrm{Bi}_{4} \mathrm{O}_{7}$. In 

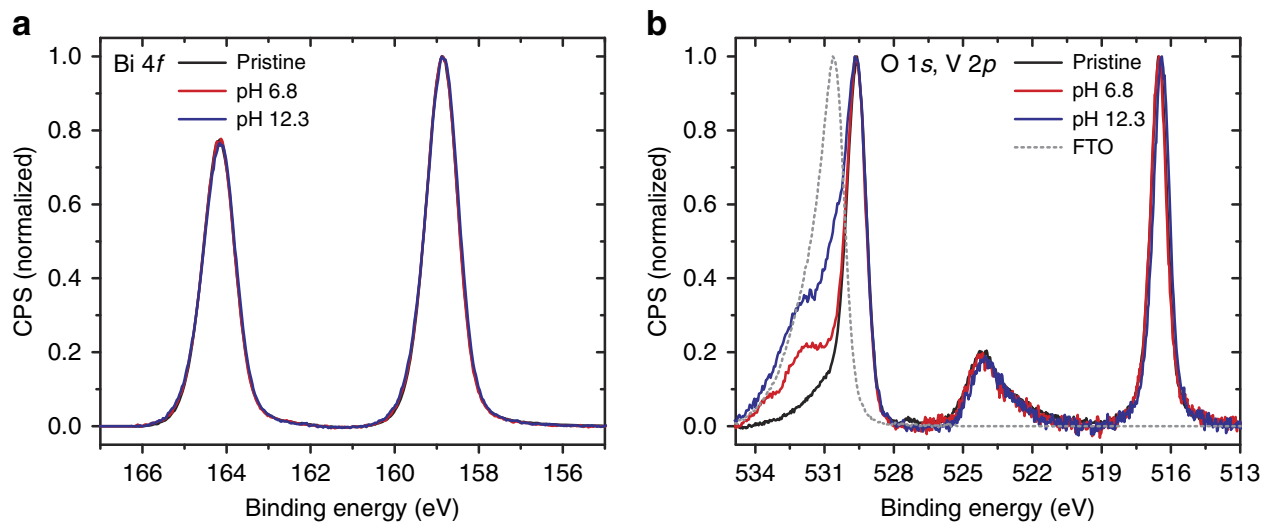

Figure 2 XPS spectra of pristine and degraded electrodes. (a) Bi $4 f_{,}$(b) $\mathrm{O} 1 \mathrm{~s}$ and $\mathrm{V} 2 p$ core levels of pristine (black) and degraded at $\mathrm{pH} 6.8$ (red) and $\mathrm{pH} 12.3$ (blue) $\mathrm{BiVO}_{4}$ samples compared with FTO (dashed grey).

addition, given that the valence band of $\mathrm{Bi}_{2} \mathrm{O}_{3}$ is energetically deeper than that of $\mathrm{BiVO}_{4}$, such a layer would be expected to introduce a barrier to photogenerated hole transport across the interface and could explain the anodic shift of the photocurrent onset potential and the reduced fill factor observed from $J-E$ curves after stability testing 22 . However, formation of a stable passivating film of bismuth oxide, such as $\mathrm{Bi}_{2} \mathrm{O}_{3}$, would be expected to impede further chemical corrosion. In contrast, we observe progressive dissolution of the films, with loss of both $\mathrm{V}$ and $\mathrm{Bi}$, as measured by RBS. In addition, similar composition results are obtained with RBS and XPS after testing the electrodes under dark conditions at an applied bias of $1.23 \mathrm{~V}$ versus RHE (Supplementary Table 1).

Determination of bismuth vanadate etching rates. Although loss of vanadium from the surface of $\mathrm{BiVO}_{4}$ in aqueous environments is consistent with prior reports, our results suggest more extensive (photo)chemical etching, with substantial loss of material from thin films. These findings prompted chemical analysis of the phosphate electrolytes used during stability tests, to detect thin film corrosion products.

Using inductively coupled plasma mass spectrometry (ICP-MS), we analysed the electrolyte solutions from samples treated in different conditions. Table 2 summarizes the estimated degradation rate $\left(\mathrm{nm} \mathrm{min}{ }^{-1}\right)$ on the basis of dissolved $\mathrm{Bi}$ and $\mathrm{V}$, calculated assuming homogeneous dissolution. These results are also shown in Fig. 3 and the complete data sets and details of calculations are provided in the Supplementary Information (Supplementary Table 2 and Supplementary Fig. 6). Under all conditions, we observe loss of both $\mathrm{Bi}$ and $\mathrm{V}$ into solution. We find that $\mathrm{BiVO}_{4}$ degradation is accelerated, in decreasing order, by light, increasing $\mathrm{pH}$ and applied bias.

To better understand the role played by solution concentration, as well as by the specific use of phosphate electrolytes, different electrolytes with different concentrations were analysed and compared with the $1 \mathrm{M}$ phosphate buffers (Fig. $3 \mathrm{~b}$ and Supplementary Information). Notably, $\mathrm{BiVO}_{4}$ thin films soaked in different electrolytes for $72 \mathrm{~h}$ tend to solubilize in all of the conditions studied. However, it is clear that the phosphate buffer concentration also plays an important role in determining the degradation rate of the material. At similar $\mathrm{pH}$ values, we observe that higher concentration accelerates degradation (Fig. 3b). Although the origin of this concentration dependence is not currently known, trends of stability with illumination, $\mathrm{pH}$ and applied bias are mostly preserved (see Supplementary Table 2). Notably, for the case of samples tested in $1 \mathrm{M} \mathrm{NaOH}(\mathrm{pH} 14)$ at $1.23 \mathrm{~V}$ versus RHE and under 1 sun AM1.5 illumination, the

\begin{tabular}{|c|c|c|c|c|c|}
\hline pH & $\begin{array}{l}\text { Light } \\
\text { condition }\end{array}$ & $\begin{array}{c}\text { Bias }^{\star} \\
\text { (V versus } \\
\text { RHE) }\end{array}$ & $\begin{array}{l}\text { Time } \\
(\min )\end{array}$ & $\begin{array}{l}\text { Degradation } \\
\text { rate on } V \text { basis } \\
\left(\mathrm{nm} \min ^{-1}\right)\end{array}$ & $\begin{array}{l}\text { Degradation } \\
\text { rate on Bi basis } \\
\left(\mathrm{nm} \mathrm{min}^{-1}\right)\end{array}$ \\
\hline \multirow[t]{4}{*}{6.8} & Light & 1.23 & 60 & 0.125 & 0.122 \\
\hline & Dark & 1.23 & 60 & 0.03 & 0.04 \\
\hline & Light & $E_{\mathrm{oc}}$ & 60 & 0.06 & 0.05 \\
\hline & Dark & $E_{\mathrm{oc}}$ & 60 & 0.1 & Not detectable \\
\hline \multirow[t]{4}{*}{12.3} & Light & 1.23 & 20 & 1.24 & 1.32 \\
\hline & Dark & 1.23 & 20 & 0.17 & 0.14 \\
\hline & Light & $E_{\mathrm{oc}}$ & 20 & 0.69 & 0.71 \\
\hline & Dark & $E_{\mathrm{oc}}$ & 20 & 0.26 & 0.21 \\
\hline
\end{tabular}

ICP-MS, inductively coupled plasma mass spectrometry, $E_{o c}$ open circuit voltage; RHE, reversible hydrogen electrode.

rate of vanadium loss from the films is significantly increased (Supplementary Fig. 6 and Supplementary Table 2). Indeed, under these conditions, we determine the etch rate on the basis of vanadium to be $2.7 \mathrm{~nm} \mathrm{~min}-1$, corresponding to the entire film thickness after $\sim 20 \mathrm{~min}$. This finding is in agreement with previous reports and is attributed to the extreme chemical instability of vanadium species under water splitting conditions at high $\mathrm{pH}(\mathrm{refs} 8,15)$.

$E x$-situ microscopy study of photochemical corrosion. The effect of photochemical degradation on the $\mathrm{BiVO}_{4}$ film morphology was determined by plan view SEM and crosssectional TEM (Fig. 4). As seen in Fig. 4a, the average grain size in as-synthesized $\mathrm{BiVO}_{4}$ films is $\sim 153 \pm 25 \mathrm{~nm}$. Following stability testing at $\mathrm{pH} 6.8$, the grains become rounded and voids form at grain boundaries, suggesting that corrosion initiates in intergrain regions of the polycrystalline films. At high $\mathrm{pH}$, rapid photocorrosion yields large regions of exposed FTO substrate, consistent with XPS observations and the higher corrosion rate determined by ICP-MS and RBS.

TEM images of pristine $\mathrm{BiVO}_{4}$ show a continuous layer of $\sim 54 \pm 13 \mathrm{~nm}$, in good agreement with the thickness determined by RBS. After stability testing at near-neutral $\mathrm{pH}$ for $1 \mathrm{~h}$, the $\mathrm{BiVO}_{4}$ layer remains largely continuous but its thickness is reduced. Inspection of the cross-sectional micrographs reveals evidence of enhanced corrosion rates near the apices and reduced corrosion rates near the valleys of the film. The overall film thickness decreases to $48 \pm 13 \mathrm{~nm}$. At high $\mathrm{pH}$, evident signs of degradation appear after stability testing for $20 \mathrm{~min}$. In apex 

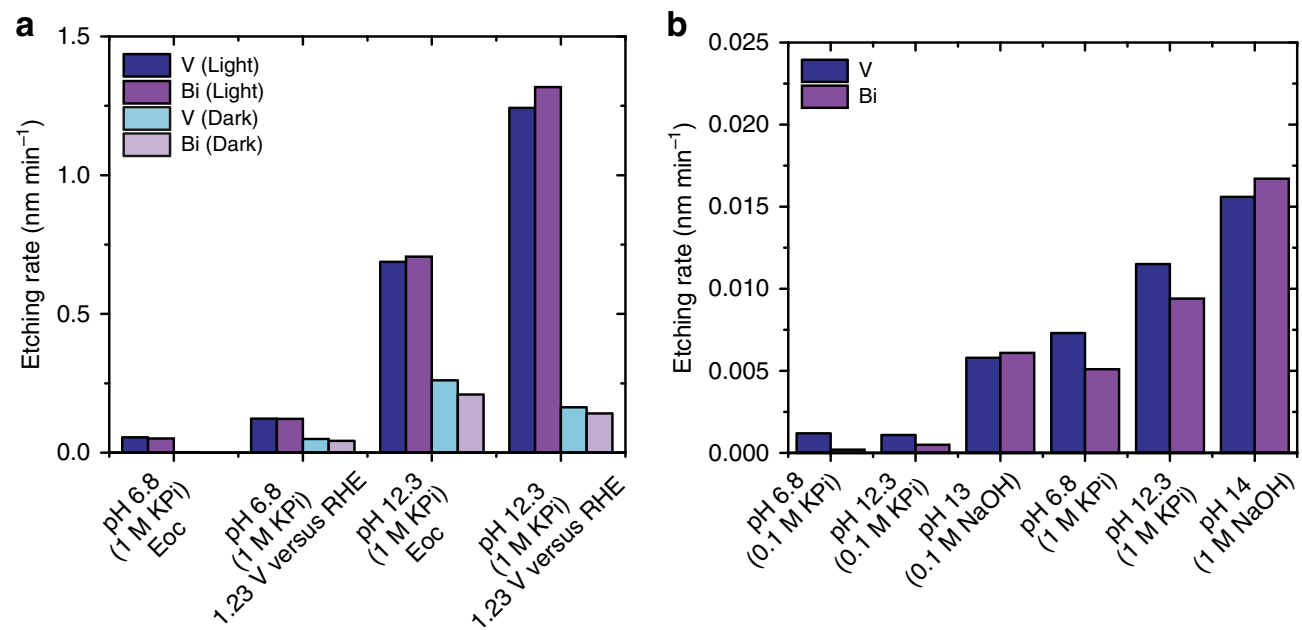

Figure 3 | Etching rates determined by ICP-MS as a function of testing conditions. (a) Etching rate on the basis of $\mathrm{V}$ (blue) and $\mathrm{Bi}$ (purple) in $\mathrm{BiVO}{ }_{4}$ substrates at different potentials and $\mathrm{pH}$ conditions under illumination and in the dark (light blue and light purple for $\mathrm{V}$ and $\mathrm{Bi}$, respectively). (b) Etching rate on the basis of $\mathrm{V}$ (blue) and $\mathrm{Bi}$ (purple) in $\mathrm{BiVO}_{4}$ substrates soaked in the dark for $72 \mathrm{~h}$ in different electrolytes at different concentrations. Homogeneous thinning of films is assumed.
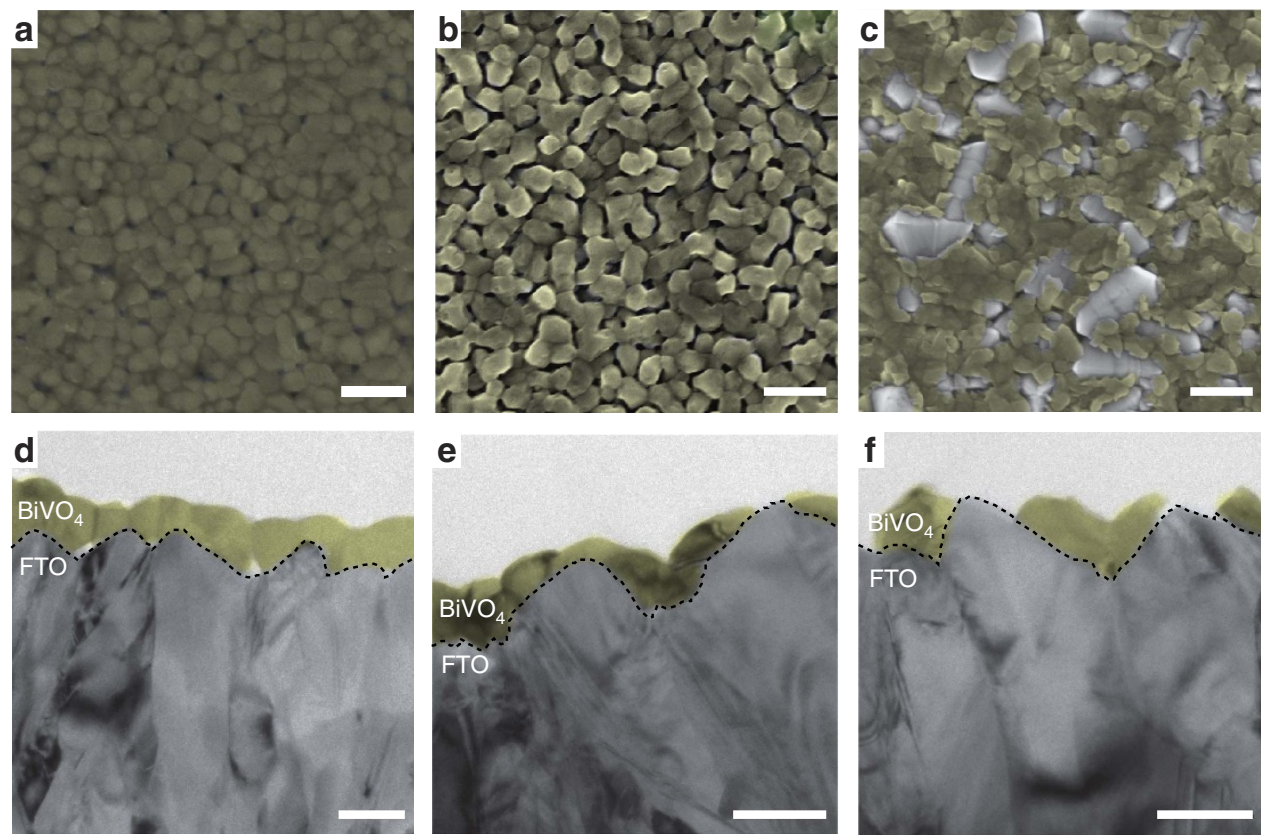

Figure 4 | SEM and TEM characterization. SEM plan view (scale bar, $500 \mathrm{~nm}$ ) and TEM cross-section (scale bar, $100 \mathrm{~nm}$ ) images of pristine (a,d), degraded at $\mathrm{pH} 6.8(\mathbf{b}, \mathbf{e})$ and at $\mathrm{pH} 12.3(\mathbf{c}, \mathbf{f}) \mathrm{BiVO}_{4}$ thin films. FTO (transparent blue) and $\mathrm{BiVO}_{4}$ (transparent yellow) are false coloured to better highlight the difference between the two layers.

regions of the substrate, complete loss of $\mathrm{BiVO}_{4}$ is observed, whereas thick islands of $\mathrm{BiVO}_{4}$ remain in valleys.

In-situ evaluation of chemical stability. Although poor stability would adversely affect the performance of an operating solar fuel device, identification and enhanced understanding of the morphological, compositional and electronic factors that contribute to corrosion is required to develop strategies for improving durability and efficiency ${ }^{23-25}$. Beyond $\mathrm{BiVO}_{4}$, such mechanistic insights are valuable for evaluating (photo)chemical susceptibilities of other transition metal oxides and approaches to designing next-generation materials that overcome present stability limitations. Therefore, to gain more information on the corrosion mechanism, in situ degradation was monitored by EC-AFM. A bias of $1.23 \mathrm{~V}$ versus RHE was applied to the $\mathrm{BiVO}_{4}$ working electrode in the electrolyte solution (1 M KPi at $\mathrm{pH} 12.3$ ) in dark, while monitoring the changes in morphology over time. A total of eight AFM topography scans were collected in the same region, to examine the degradation process over a period of $160 \mathrm{~min}$ (Fig. 5a,b and Supplementary Movie 1). Specifically, three different regions within the same scanned area (Fig. 5a) were selected for evaluation of variations in height and surface-tovolume ratio (Fig. 5c,d), as well as the distribution of the height profile. Close inspection of these images reveals that degradation occurs approximately uniformly at crystallographic facets exposed to the electrolyte.

Region 1 exhibits three surfaces (solid/liquid interfaces) in the upper, side and bottom part of the frame that are exposed to the electrolyte. Notably, etching progresses approximately uniformly from all three solid/liquid interfaces. In addition, the height histogram from Region 1 reveals that simultaneous etching occurs 
from the exposed top surface. In contrast, Region 2 has just one solid/liquid interface, which runs diagonally across the upper left portion of the region, exposed directly to electrolyte. Importantly, solid/solid boundaries that are present within this region do not appear to be specific sites for chemical attack. Analysis of the corresponding height histogram supports this interpretation, in which an approximately bimodal distribution is observed, with low and high values corresponding to the substrate and the $\mathrm{BiVO}_{4}$ grain, respectively. Region 3 exhibits no solid/liquid interfaces and is instead characterized by the presence of solid/solid grain boundaries, along with the exposed top surface of the central grain. Thus, this region is more protected from chemical attack and corrosion occurs almost exclusively from the top surface. As corrosion propagates from the top surface, the distribution of heights shifts monotonically towards smaller values.

Based on these in-situ EC-AFM measurements, we can conclude that corrosion of the material initiates predominantly from solid/liquid interfaces. We find no indication for selective etching of localized regions, such as at secondary phases at grain boundaries, or undercutting of the film at its interface to the FTO substrate. However, as these polycrystalline films are not completely compact, the evolution of morphology depends strongly on local geometry. Voids in intergrain regions act as sites for more rapid chemical attack, whereas adjacent grains in intimate physical contact with one another are partially protected. For those regions that are characterized by multiple solid/liquid interfaces, increases of the microscopic surface to volume ratio as etching progresses could lead to accelerated corrosion (Fig. 5d). We note that further advancement of the EC-AFM, currently underway in our laboratories, will allow for implementation of light illumination of the sample under operating conditions. However, the current time-lapse testing procedure enabled in situ monitoring of changes over a time scale compatible with the scan rate used for EC-AFM (Supplementary Movie 1).

Computational evaluation of (photo)chemical corrosion. Computational methods are employed to interpret our experimental results and they provide insight into the mechanism of corrosion under dark and illuminated conditions. We first consider the thermodynamic stability of $\mathrm{BiVO}_{4}$ under dark conditions at the open circuit potential, where we observe slow but progressive dissolution of films at all $\mathrm{pH}$ values. To this end, we have calculated Bi-V Pourbaix diagrams with ion concentrations of $10^{-5} \mathrm{~mol} \mathrm{~kg}^{-1}$, as implemented in the Materials Project $^{26}$ using the hybrid calculated-experimental Pourbaix formalism approach based on the work of Persson et al. ${ }^{27}$. To the best of our knowledge, an experimental Pourbaix diagram for $\mathrm{BiVO}_{4}$ has not been established. However, we can gain insight into the processes responsible for photochemical and chemical corrosion of $\mathrm{BiVO}_{4}$ by considering equilibrium thermodynamic stability against decomposition into any combination of solid and/or aqueous species. As shown in Fig. $6 \mathrm{a}, \mathrm{BiVO}_{4}$ is stable in a large region with $\mathrm{pH}$ values from 1 to 11 and at the electrochemical potentials within the water stability region. a

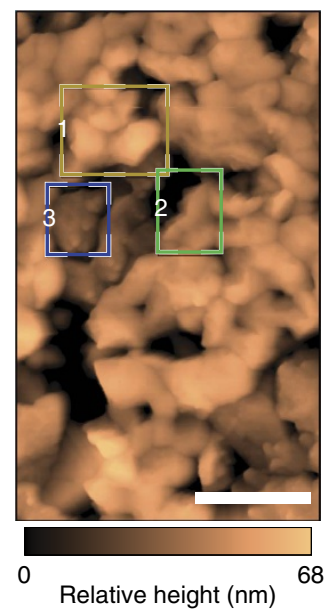

c

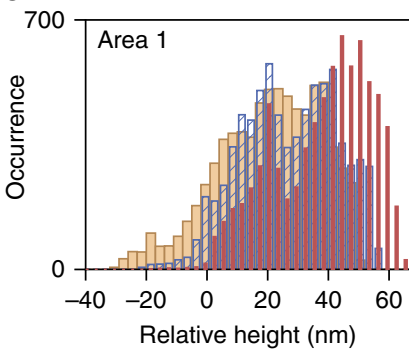

b
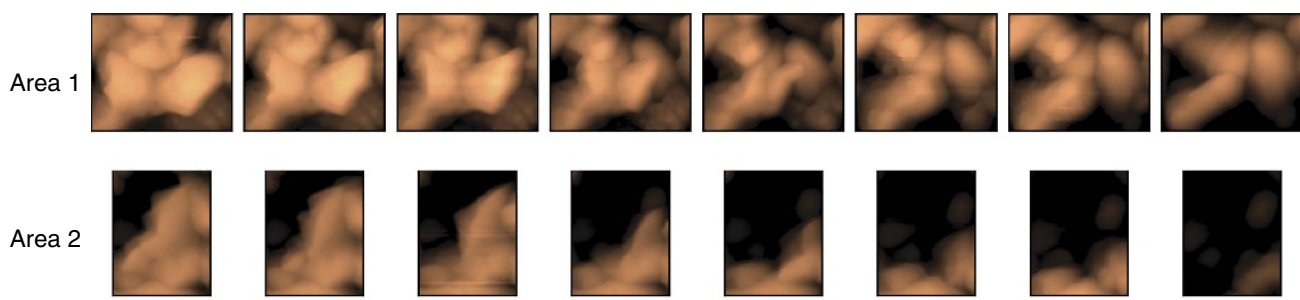

Area 3
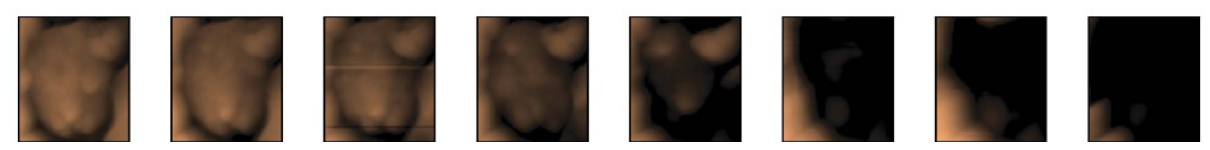

0

Time (min)
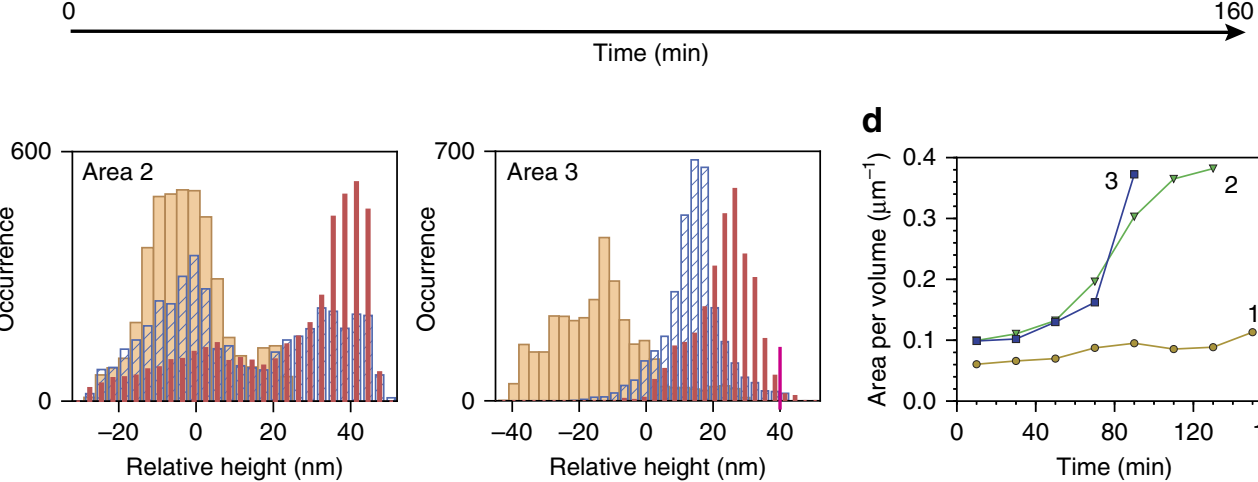

d

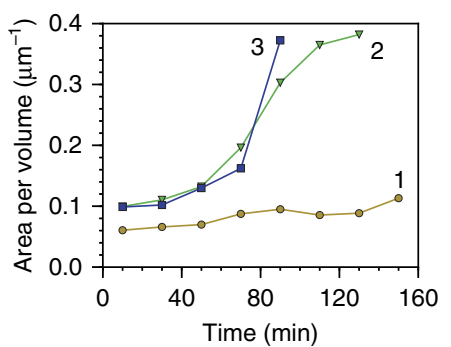

Figure 5 | Monitoring corrosion via in situ EC-AFM. (a) EC-AFM scan $\left(527 \times 898 \mathrm{~nm}^{2}\right.$; scale bar, $\left.200 \mathrm{~nm}\right)$. The reported height is relative to a point on the underlying FTO substrate that is resolvable in all EC-AFM images. Common points have been used to align eight subsequent $1 \times 1 \mu \mathrm{m}^{2}$ scans of the same region, to correct for sample drift and rotation. Three coloured boxes indicate Regions 1 (yellow), 2 (green) and 3 (blue), whose temporal evolution were tracked in detail. (b) Aligned EC-AFM scans in the three regions indicated in a were used to monitor corrosion-induced changes to BiVO 4 morphology at 20 min increments in $1 \mathrm{M} \mathrm{KPi} \mathrm{(} \mathrm{pH}$ 12.3). (c) Histograms showing height distributions in each of the three regions at the 10 (red)-, 70 (blue)and 150 (orange)-min marks demonstrate that the heights of the $\mathrm{BiVO}_{4}$ film decreases over time. Height values <0 nm correspond to the underlying FTO substrate. (d) Progression of the surface area to volume ratio for region 1 (yellow), 2 (green) and 3 (blue) over the course of the 160-min test. 
Unfortunately, at potentials close to the oxygen evolution potential, $\mathrm{BiVO}_{4}$ is no longer thermodynamically stable. Its constituent $\mathrm{V}$ is expected to preferentially dissolve as $\mathrm{VO}_{4}^{-}$ions. This is consistent with the single-element V Pourbaix diagram, where solid phases are dissolved and only $\mathrm{VO}_{4}^{-}$ions exist in the same region.

From a thermodynamic perspective, it is expected that the $\mathrm{BiVO}_{4}$ would self-passivate via formation of a chemically stable bismuth oxide at its surface. However, although our XPS experiments indicate some $\mathrm{Bi}$ enrichment at the surface following extended dark stability testing, we also detect the presence of $\mathrm{Bi}$ ions in the supporting electrolyte and conclusively observe that bulk dissolution of the $\mathrm{BiVO}_{4}$ occurs and self-passivation does not impede corrosion. This experimental result indicates that the thermodynamic equilibrium between the solid and liquid is not established. Indeed, a self-passivated surface of $\mathrm{BiVO}_{4}$ would require formation of a more stable $\mathrm{Bi}-\mathrm{O}$ surface phase (for example, $\mathrm{Bi}_{4} \mathrm{O}_{7}$ as seen at higher potentials and $\mathrm{pH}$ ). However, the vanadium to bismuth content in $\mathrm{BiVO}_{4}$ is $1: 1$, which means that $50 \%$ of the cations will dissolve, rendering the resulting $\mathrm{Bi}-\mathrm{O}$ framework highly defective and unstable. Formation of a stable bismuth oxide or hydroxide would require significant structural rearrangement, which may be kinetically hindered under roomtemperature aqueous conditions. Given these considerations, we can visualize the scenario in which structural rearrangement to form a passivating oxide on the solid surface is kinetically unfavourable by artificially removing all solid $\mathrm{Bi}$-oxide phases above the OER potential (in this case, $\mathrm{Bi}_{4} \mathrm{O}_{7}$ and $\mathrm{Bi}_{2} \mathrm{O}_{3}$ ) from the set of phases included in the construction of the Pourbaix diagram. The result is shown in Fig. 6b, where we observe that only ions exist in the aqueous solution in the entire $\mathrm{pH}$ range above the OER potential. This result reveals that thermodynamic considerations cannot describe the observed dark stability of $\mathrm{BiVO}_{4}$ thin films. Rather, kinetic limitations associated with a

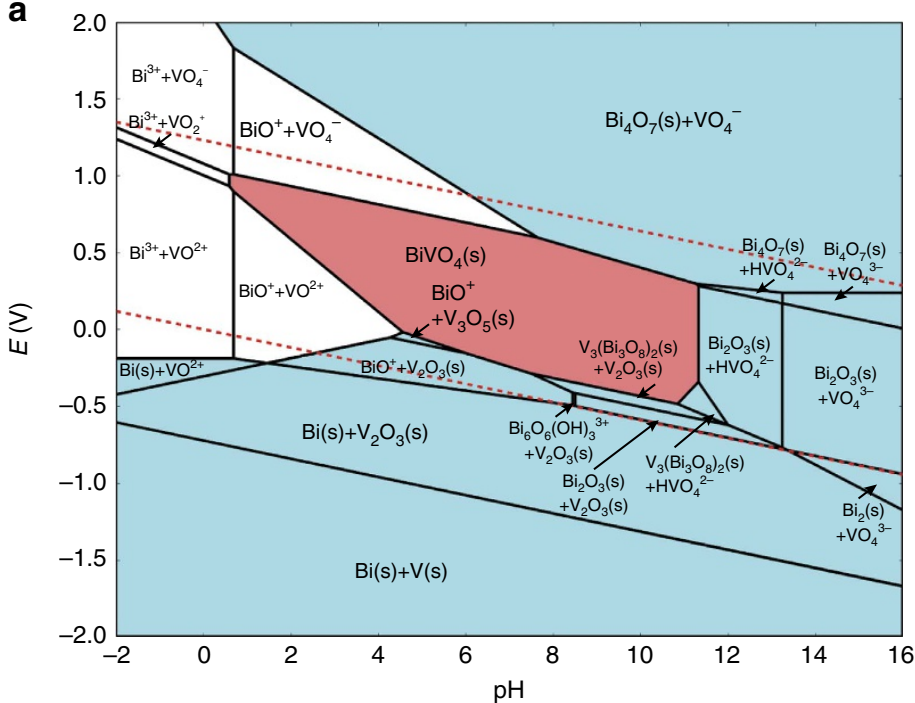

b

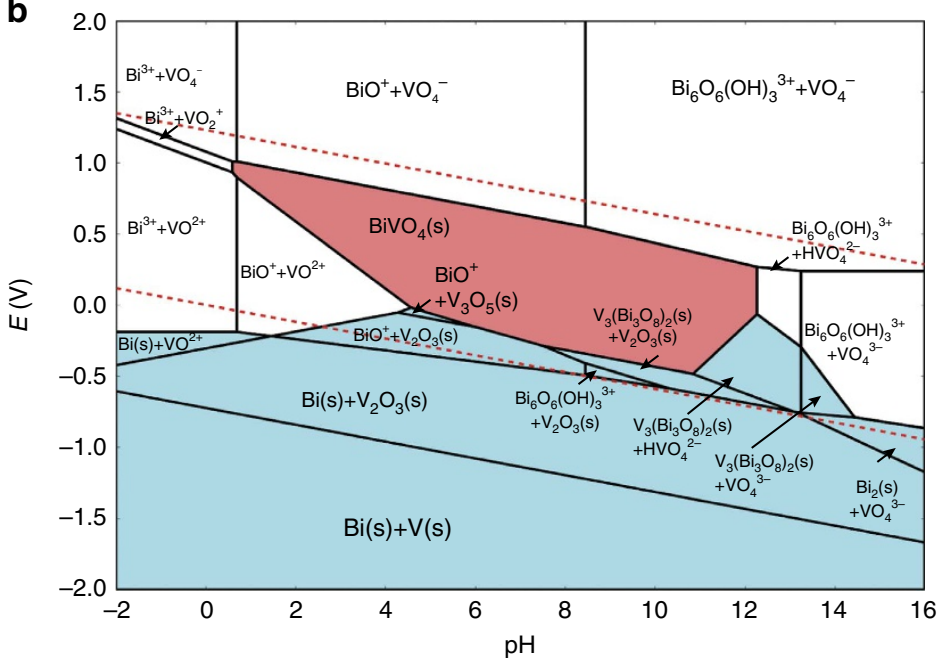

C

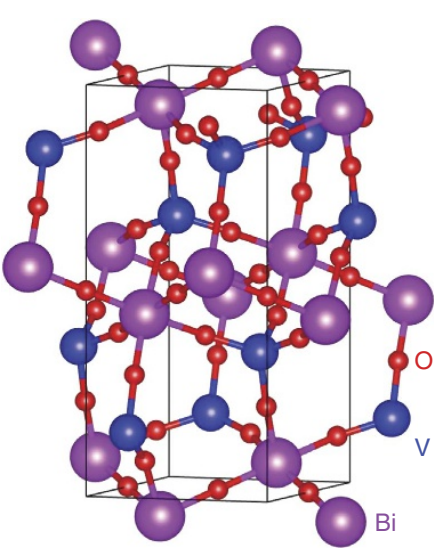

d

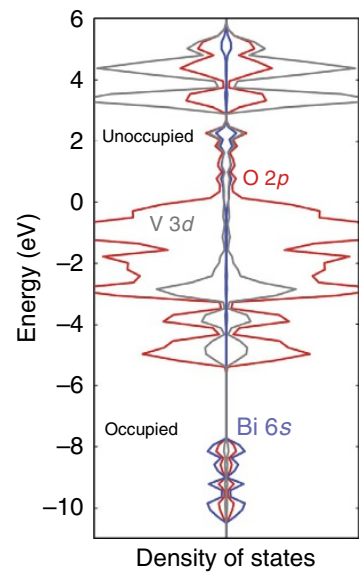

Figure 6 | Evaluation of illuminated and dark stability by computational methods. (a) The Materials Project Pourbaix diagram of 50-50\% Bi- $\mathrm{V}$ system in aqueous solution, assuming a $\mathrm{Bi}$ ion concentration at $10^{-5} \mathrm{~mol} \mathrm{~kg}-1$ and a $\mathrm{V}$ ion concentration at $10^{-5} \mathrm{~mol} \mathrm{~kg}^{-1}$, and (b) a Pourbaix diagram with $\mathrm{Bi}_{2} \mathrm{O}_{3}$ and $\mathrm{Bi}_{4} \mathrm{O}_{7}$ phases removed. The upper red dashed line represents the potential for the oxygen evolution reaction (OER), whereas the lower red dashed line corresponds to the potential for the hydrogen evolution reaction (HER). The cyan regions denote stable solid compounds, while the pink region is $\mathrm{BiVO}_{4}$. In the white regions, only ions are stable in aqueous solution. (c) Illustration of predicted $\mathrm{BiVO}_{5}$ structure where blue, magenta and red spheres denote the $\mathrm{V}, \mathrm{Bi}$ and $\mathrm{O}$ atoms, respectively. Thermodynamic evaluation of the stability of this hypothetical compound, which consists of lattice $\mathrm{Bi}^{5+}$ and $\mathrm{V}^{5+}$, is used to predict the effect of surface accumulation of photogenerated holes in $\mathrm{BiVO}_{4}$. (d) Atom projected density of states of BiVO 5 . The energy of the Fermi level is set to $0 \mathrm{eV}$. 
room-temperature structural rearrangements of corrosion products must be considered. Such a mechanism for kinetically inaccessible self-passivation is expected to be increasingly important for complex, multi-component oxides. Computational evaluation of possible kinetically hindered surface transformations may be essential for predicting the real world stabilities of novel compounds and for designing strategies for overcoming chemical instabilities.

In addition to the dark chemical instability of $\mathrm{BiVO}_{4}$ thin films, our experimental results indicate that dissolution of the material in water is accelerated by illumination, as well as anodic biasing to $1.23 \mathrm{~V}$ versus RHE. These findings suggest that accumulation of photogenerated holes at the surface of $\mathrm{BiVO}_{4}$ promotes photocorrosion. Furthermore, we note that previous studies have found that integration of a catalyst on the surface can suppress photocorrosion and enhance long-term stability of $\mathrm{BiVO}_{4}$ (refs 8,15,16,19,28-31), even for the case of porous and ion-permeable catalysts, such as $\mathrm{CoPi}$ and $\mathrm{FeO}(\mathrm{OH})$, which do not physically isolate the semiconductor from the electrolyte environment. This suggests that efficient extraction of photogenerated holes from the near-surface region reduces chemical attack of the material.

Given these observations of photoinduced chemical instability, we consider the role of surface-accumulated holes under photoexcitation, as well as the strongly oxidative conditions present with the production of reactive oxygen species (for example, $\mathrm{OH}^{\bullet}$ and $\left.\mathrm{O}^{-}\right)^{32,33}$, on the stability of $\mathrm{BiVO}_{4}$. The valence band maximum, where photogenerated holes accumulate, consists of considerable $\mathrm{Bi} 6 s$ orbital character, hybridized with O $2 p$ (ref. 7). In prior theoretical work, Kweon and Hwang ${ }^{34,35}$ showed that large polaron holes are the stable positive charge carriers within the monoclinic phase of this material, and that, at the surface, holes localize to $\mathrm{BiO}_{6}$ polyhedra, with $22 \%$ of hole charge density on $\mathrm{Bi}$ cations and an average of $8 \%$ of hole charge density on each of the six $\mathrm{O}$ anions in the polyhedron, with balance of charge located outside of the polyhedron. Therefore, we first consider the impact of the modified charge density around $\mathrm{Bi}$ ions due to hole localization on stability. To this end, we hypothesize the scenario that brings $\mathrm{Bi}^{3+}$ to $\mathrm{Bi}^{5+}+2 \mathrm{e}^{-}$in $\mathrm{BiVO}_{4}$, as $\mathrm{Bi}^{5+}$ is a potentially stable oxidation state for $\mathrm{Bi}$, whereas $\mathrm{Bi}^{4+}$ is uncommon ${ }^{36,37}$. To obtain a plausible structure representing a $\mathrm{Bi}^{5+} / \mathrm{V}^{5+}$ compound or film, we use the Materials Project structure predictor ${ }^{38,39}$ to predict a new oxide compound containing only $\mathrm{Bi}^{5+}$ and $\mathrm{V}^{5+}$ valence states. By calculating the stability of an optimized bulk $\mathrm{Bi}^{5+}$ and $\mathrm{V}^{5+}$ oxide, we can obtain a qualitative picture of the stability of such a configuration in an oxide framework. The structure predictor algorithm searches for the most suitable structure among all known structures found in the $\operatorname{ICSD}^{40}$ for the target ions and valence states (in this case, $\mathrm{Bi}^{5+}$ and $\mathrm{V}^{5+}$ ) through a datamining approach ${ }^{38,39}$. The resulting structure is shown in Fig. $6 \mathrm{c}$. From our calculations, the projected density of states show that the $\mathrm{Bi} 6 s$ orbitals are very strongly hybridized with the $\mathrm{O} 2 p$ orbitals, both in the valence band and conduction band (Fig. 6d). Considering that the magnetic moment is zero in the system, it is confirmed that $\mathrm{Bi}$ and $\mathrm{V}$ in $\mathrm{BiVO}_{5}$ are both in $5+$ oxidation states. A new phase diagram for the $\mathrm{Bi}-\mathrm{V}-\mathrm{O}$ system is generated (Supplementary Fig. 7) and it is found that $\mathrm{BiVO}_{5}$ is unstable by $0.215 \mathrm{eV}$ per atom towards oxygen release. This large above-hull energy, defined as the energy of unstable phases, strongly indicates that the thermodynamic driving force for $\mathrm{BiVO}_{5}$ to decompose into $\mathrm{BiVO}_{4}$ and $1 / 2 \mathrm{O}_{2}$ is significant. One of the reasons for the instability of $\mathrm{BiVO}_{5}$ is that the small ionic radius of $\mathrm{Bi}^{5+}$ is not compatible with the $\mathrm{BiO}_{8}$ structure motif in the crystal. These results suggest that any photo-induced oxidation event on $\mathrm{Bi}$ is likely to further destabilize the compound and increase the rate of corrosion under operating conditions, due to an effective $\mathrm{Bi}-\mathrm{O}$ bond strain in the presence of $\mathrm{Bi}^{5+}$.

In addition to hole localization on $\mathrm{Bi}$ ions, we also consider the impact of oxidation of $\mathrm{O}^{2-}$ to $\mathrm{O}^{-}$at the surface of $\mathrm{BiVO}_{4}$. Such a process has been implicated in the photocorroison mechanism of, for example, $\mathrm{ZnO}$, where it has been proposed that degradation proceeds through hole trapping at oxygen surface sites to yield formation of $\mathrm{O}^{-}$intermediate that results in irreversible loss of oxygen from the lattice as $\mathrm{O}_{2}$ and consequent release of $\mathrm{Zn}^{2+}$ ions in the solution ${ }^{33}$. Although the charge density of surfaceaccumulated holes has been predicted to be higher at Bi cations than $\mathrm{O}$ anions, such a mechanism is not precluded. Furthermore, the energetic position of the valence band maximum of $\mathrm{BiVO}_{4}$, at $\sim 7.27 \mathrm{eV}$ below the vacuum level, renders formation of radical $\mathrm{OH}^{\bullet}$ species, as found by Bard and colleagues ${ }^{33}$. Participation of such radicals in the oxygen loss processes described above may serve to further accelerate photocorrosion, in particular at high $\mathrm{pH}$.

\section{Discussion}

We demonstrate that polycrystalline $\mathrm{BiVO}_{4}$ thin films are susceptible to chemical and photochemical corrosion. Indeed, chemical attack of the semiconductor is observed under all aqueous testing conditions and is accelerated by illumination, increasing $\mathrm{pH}$ and applied anodic bias. Although RBS reveals near-stoichiometric composition in the bulk of as-deposited thin films, XPS indicates a non-stoichiometric composition at the surface that is affected by exposure to electrolyte. Observation of film dissolution in real time by EC-AFM indicates that chemical attack propagates approximately uniformly from exposed solid/liquid interfaces. Therefore, there is no indication of preferential chemical attack at specific regions or at crystal facets of grains, but consumption rate is dependent on local solid/liquid interface morphology. In contrast to thermodynamic predictions, self-passivation of $\mathrm{BiVO}_{4}$ by the formation of a chemically stable Bi oxide surface phase is not observed and bulk dissolution of films occurs on extended exposure to aqueous electrolytes. We attribute the bulk chemical instability to kinetic factors that limit room-temperature structural transformation of the $\mathrm{V}$-deficient degradation product into a stable $\mathrm{Bi}$ oxide phase. In addition, photoexcitation results in accumulation of holes at the surface of $\mathrm{BiVO}_{4}$ that destabilize the lattice and increase the dissolution rate.

Mechanistic insights into the chemical and photochemical instability of $\mathrm{BiVO}_{4}$ can be used to guide approaches to stabilization and aid the search for active catalysts and functional interfaces that can improve durability for sustained operation. For example, efficient transfer of holes to catalyst on the surface will dramatically reduce the corrosion rate. However, unless this catalyst layer is dense and impermeable, long-term durability may be limited by the native dark chemical stability of the material and self-passivation cannot be assumed. We note that introduction of surface layers that reduce the corrosion rate and enable time for structural reorganization of the surface before dissolution might promote self-passivation and kinetically stabilize the material. Beyond $\mathrm{BiVO}_{4}$, the methodology presented here can be applied for rigorous investigation of the stabilities of new and emerging functional materials under operating conditions. Identification of kinetic limitations to self-passivation points to a new set of factors that should be considered in the experimental and computational search for stable, visible-light-absorbing semiconductors for photoelectrochemical energy conversion.

\section{Methods}

Fabrication of $\mathrm{BiVO}_{4}$ thin film photoelectrodes. Spin-coated bismuth vanadate thin films were prepared using a procedure adapted from the literature ${ }^{6}$. FTO films with nominal resistivity of $\sim 13 \Omega / \square$ (Sigma Aldrich) on $10 \times 10 \mathrm{~cm}^{2}$ glass 
substrates were thoroughly washed with isopropanol, detergent (Alconex) in deionized water and pure deionized water, dried with a nitrogen gun and treated for $10 \mathrm{~min}$ with an ozone cleaner (Jelight Model 42) before deposition of $\mathrm{BiVO}_{4}$ by spin coating. In a typical deposition, $15 \mathrm{ml}$ of a $0.2 \mathrm{M}$ solution of bismuth (III) nitrate pentahydrate (Sigma Aldrich, $\geq 98 \%$ ) in acetylacetone (Sigma Aldrich, $\geq 99 \%$ ) and $100 \mathrm{ml}$ of a $0.03-\mathrm{M}$ solution of vanadium(IV)-oxy acetylacetonate in acetylacetone were prepared separately and sonicated for $10 \mathrm{~min}$. Then, the two solutions were mixed together and sonicated for an additional $5 \mathrm{~min}$.

Approximately 1-1.2 millilitres of the resulting solution millilitres (enough solution to homogeneously cover the whole surface) was filtered with $0.45 \mu \mathrm{m}$ nylon filters (Thermo Fisher Scientific) and dispensed onto the $10 \times 10 \mathrm{~cm}^{2} \mathrm{FTO} /$ glass slide. The substrate was then spun twice in a row at 1,000 r.p.m. for $6 \mathrm{~s}$ on a spin coater (Laurell Technologies) with an acceleration rate of 150 r.p.m. $\mathrm{s}^{-1}$. After this spin-coating cycle, the substrate was annealed for $10 \mathrm{~min}$ in air at $500^{\circ} \mathrm{C}$ in a muffle furnace (Cole-Parmer). This procedure (that is, spin coating followed by short annealing) was repeated nine times. After the last spin-coating cycle, the substrate was annealed for $2 \mathrm{~h}$ at $500^{\circ} \mathrm{C}$ to achieve a final thickness of $\sim 50 \mathrm{~nm}$

$\mathrm{BiVO}_{4}$ thin film photoelectrodes were prepared by dicing the $\mathrm{BiVO}_{4}$-coated FTO/glass into $\sim 1 \mathrm{~cm} \times 1.3 \mathrm{~cm}$ pieces. To expose FTO to provide electrical connection to external leads, an $\sim 0.3$-cm-wide strip of the $\mathrm{BiVO}_{4}$ thin film along the long side of the electrode was etched away using $0.1 \mathrm{M} \mathrm{HCl}$ (Sigma Aldrich, ACS reagent, 37\%). A Cu wire was connected to the exposed FTO using silver conductive epoxy (Circuit Works, CW2400) and the electrode with the wire was dried for $20 \mathrm{~min}$ at $60^{\circ} \mathrm{C}$. Then, the wire was isolated with a glass tube and the $\mathrm{Cu}$ wire, silver epoxy and exposed FTO were all sealed together with the glass tube using a non-conductive aqueous resistant epoxy (Loctite $615 \mathrm{Hysol}$ ). Exposed surface areas of the assembled photoelectrodes were determined by optical scanning and digital image analysis with Image J. Typical areas were in the range of $0.5-1.0 \mathrm{~cm}^{2}$.

Photoelectrochemistry and stability measurements. All photoelectrochemical measurements were performed in a three-electrode photoelectrochemical cell with a planar quartz window $\left(5 \mathrm{~cm}^{2}\right)$ using a BioLogic SP200 potentiostat. Bismuth vanadate photoanodes were configured as the working electrodes, a coiled Pt wire was used as counter electrode and a $\mathrm{Ag} / \mathrm{AgCl}(3 \mathrm{M} \mathrm{NaCl}$, BASI) served as reference electrode. Measurements were performed in electrolyte consisting of $1 \mathrm{M} \mathrm{KPi}$ buffer solutions at $\mathrm{pH} 6.8$ or 12.3 with or without $0.1 \mathrm{M}$ sodium sulfite as sacrificial reagent, depending on the particular experiment. In detail, $1 \mathrm{M} \mathrm{KPi}$ at $\mathrm{pH} 6.8$ was prepared from KPi monobasic (Sigma Aldrich, $\geq 99 \%, 34.0 \mathrm{~g}, 0.25 \mathrm{~mol}$ ) and $\mathrm{KPi}$ dibasic (Sigma Aldrich, $\geq 98 \%, 43.5 \mathrm{~g}, 0.25 \mathrm{~mol}$ ) in $500 \mathrm{ml}$ of water. Likewise, $1 \mathrm{M}$ $\mathrm{KPi}$ at $\mathrm{pH} 12.3$ was prepared using KPi dibasic $(43.5 \mathrm{~g}, 0.25 \mathrm{~mol}$ ) and $\mathrm{KPi}$ tribasic (Sigma Aldrich, $\geq 98 \%, 53.1 \mathrm{~g}, 0.25 \mathrm{~mol}$ ) in $500 \mathrm{ml}$ of water. The diffusion constants for $\mathrm{HPO}_{4}^{2-}$ and $\mathrm{PO}_{4}^{3-}$ are reported in Supplementary Note 1 (ref. 41). To evaluate the performance of photoanodes as semiconductor light absorbers, sodium sulfite (Sigma Aldrich, $\geq 98 \%, 6.3 \mathrm{~g}, 0.05 \mathrm{~mol}$ ) was added to buffer solutions as a sacrificial hole acceptor. For material degradation studies, measurements were performed in the absence of sodium sulfite. Unless otherwise noted, all measurements under illumination were performed using simulated AM 1.5 light (Solar Light) adjusted to $100 \mathrm{~mW} \mathrm{~cm}^{-2}$ using a calibrated Si photovoltaic cell (SolarSim calibration, Newport).

Systematic evaluations of performance and stability at $\mathrm{pH} 6.8$ and 12.3 were carried out using the following procedure. First, baseline photoelectrochemical performance was established by running CVs from the open circuit potential $\left(E_{\mathrm{oc}}\right)$ to $1.8 \mathrm{~V}$ versus RHE at a scan rate of $100 \mathrm{mV} \mathrm{s}^{-1}$ in the presence of $0.1 \mathrm{M}$ sodium sulfite as sacrificial reagent. Both dark and illuminated CVs were collected. It is noteworthy that for the sake of clarity, only anodic sweeps obtained from these CVs are shown in Fig. 1. Complete CVs are shown in Supplementary Fig. 4. Second, stability of the same electrodes was tested by performing chronoamperometry in pure $1 \mathrm{M} \mathrm{KPi}$ buffer solutions ( $\mathrm{pH} 6.8$ or 12.3) in the dark or under illumination at $E_{\mathrm{oc}}$ or at $1.23 \mathrm{~V}$ versus RHE. Fresh electrodes were used for each of the illumination and $\mathrm{pH}$ conditions. Stability tests at $\mathrm{pH} 6.8$ were run for $60 \mathrm{~min}$, whereas those at $\mathrm{pH} 12.3$ were run for $20 \mathrm{~min}$ due to the more rapid degradation. Finally, the post-degradation performance was evaluated by collecting CVs using conditions identical to those used for baseline performance evaluations.

Material characterization. Baseline X-ray diffraction characterization of FTO/glass substrate coated with a spin-cast $\mathrm{BiVO}_{4}$ thin film was obtained with a Rigaku SmartLab X-ray diffractometer. X-ray diffraction patterns were collected with a $\mathrm{Cu}$ source at $0.5^{\circ}$ with respect to the sample and parallel beam optics. To provide insights into the mechanism and consequences of $\mathrm{BiVO}_{4}$ degradation under photoelectrochemical conditions, comparative measurements before and after stability testing were performed using a variety of complementary spectroscopic and microscopic methods. To evaluate morphological changes, SEM was performed on a Zeiss Gemini Ultra-55 analytical field emission SEM and on an FEI Quanta 250 FEG. In addition, cross-sectional TEM images were obtained on an FEI Tecnai F30, with a $300-\mathrm{kV}$ acceleration voltage.

The near-surface chemical composition and valence band structure were determined by XPS using a monochromatized $\mathrm{Al} \mathrm{K} \alpha$ source $(\mathrm{h} v=1486.6 \mathrm{eV})$, operated at $225 \mathrm{~W}$, on a Kratos Axis Ultra DLD system at a takeoff angle of $0^{\circ}$ relative to the surface normal and a pass energy for the narrow scan core level and valence band spectra of $20 \mathrm{eV}$. Spectral fitting was conducted using Casa XPS analysis software. Spectral positions were corrected using adventitious carbon by shifting the $\mathrm{C} 1 s$ core level position to $284.8 \mathrm{eV}$ and curves were fit with quasi-Voigt lines following Shirley background subtraction.

Complimentary bulk composition measurements were obtained by RBS, which also allowed for determination of nominal film thickness. $\mathrm{He}^{+}$ions were accelerated to $3,040 \mathrm{keV}$ on a $5 \mathrm{SDH}$ pelletron tandem accelerator manufactured by National Electrostatics Corporation with a sample tilt of $30-60^{\circ}$ and backscattered ions were detected at an angle of $165^{\circ}$ using a Si surface barrier detector. Data fitting was performed with SIMNRA software. RBS analysis may allow for calculation of an effective film thickness, assuming a planar and homogeneous $\mathrm{BiVO}_{4}$ overlayer deposited on a flat surface. Although our films exhibit good coverage with a reduced roughness on the surface, the underlying FTO has a similar roughness and we have to account that the model is affected by these parameters.

Analysis of corrosion products in electrolyte solution was conducted by ICP-MS with an Agilent 7,900 using a standard configuration consisting of Micromist nebulizer and quartz sample introduction system of Scott-type with spray chamber and one piece torch with a $2.5-\mathrm{mm}$ inner diameter injector; $\mathrm{Ni}$ cones were used throughout. Internal standards were added on-line via the sample delivery peristaltic pump. The instrument was optimized using default autotune conditions directly from the MassHunter Workstation for U-HMI. Samples were measured in helium collision mode for all analytes.

EC-AFM was performed on a Bruker Dimension Icon AFM system, using an in situ electrochemical cell as sample holder, equipped with a $\mathrm{BiVO}_{4}$ working electrode, a coiled Pt wire as counter electrode and a $\mathrm{Ag}$ wire as pseudo-reference. All the measurements were performed in $\mathrm{pH} 12.3$ and a BioLogic SP200 potentiostat was used to apply a $1.23 \mathrm{~V}$ versus RHE bias. Topography AFM images were acquired in Scanasyst mode with Si tips (Bruker Scanasyst-Air). It is worth noting that no degradation of the Si tip was observed over the measurement period.

Evaluation of illuminated and dark stability by theoretical methods. The firstprinciples calculations are based on density functional theory using the Vienna software package $(\mathrm{VASP})^{42}$. We choose the PAW pseudopotentials ${ }^{43}$ with the generalized gradient approximation as implemented by Perdew, Burke and Ernzerhoff $\left(\mathrm{PBE}^{44}\right)$. The PBE $+\mathrm{U}$ method is used to address the on-site Coulomb interactions in the localized $d$ orbitals by adding an additional Hubbard-type $\mathrm{U}$-term with the value of $3.25 \mathrm{eV}$ for $\mathrm{V}$ as reported for binary metal oxides ${ }^{45}$. A $6 \times 6 \times 6$ Monkhorst-Pack $k$-point mesh for the integrations over the Brillouin zone is used. The energy cutoff is $450 \mathrm{eV}$ and the spin polarization is included in all calculations

The analysis of the Bi-V ternary Pourbaix diagram is based on the work of Persson et al. ${ }^{46}$, where solid and dissolved species are combined in a single-phase diagram to determine the stable species (solids and/or aqueous ions) as a function of $\mathrm{pH}$ and potential ${ }^{36}$. Following ref. 46, the theoretical data obtained with the $\mathrm{PBE}+\mathrm{U}$ method are used for the total energies of solid phases and the experimental data are used for the dissolved ions.

To construct the phase diagram, we use the phase diagrams app ${ }^{47}$ from the Materials Project database. By calculating the energies of all known compounds in a given chemical system (for example, $\mathrm{Bi}-\mathrm{V}-\mathrm{O}$ ), we can determine the phase diagram for that system at $0 \mathrm{~K}$ and $0 \mathrm{~atm}$.

Data availability. The source data that support the findings of this study are included in the Article, Supplementary Information and Supplementary Data 1.

\section{References}

1. Maeda, K. \& Domen, K. Photocatalytic water splitting: recent progress and future challenges. J. Phys. Chem. Lett. 1, 2655-2661 (2010).

2. McKone, J. R., Lewis, N. S. \& Gray, H. B. Will solar-driven water-splitting devices see the light of day? Chem. Mater. 26, 407-414 (2013).

3. Sivula, K. Metal oxide photoelectrodes for solar fuel production, surface traps, and catalysis. J. Phys. Chem. Lett. 4, 1624-1633 (2013).

4. Chen, S. \& Wang, L.-W. Thermodynamic oxidation and reduction potentials of photocatalytic semiconductors in aqueous solution. Chem. Mater. 24, 3659-3666 (2012).

5. Park, Y., McDonald, K. J. \& Choi, K.-S. Progress in bismuth vanadate photoanodes for use in solar water oxidation. Chem. Soc. Rev. 42, 2321-2337 (2013).

6. Cooper, J. K. et al. Indirect bandgap and optical properties of monoclinic bismuth vanadate. J. Phys. Chem. C 119, 2969-2974 (2015).

7. Cooper, J. K. et al. Electronic structure of monoclinic $\mathrm{BiVO}_{4}$. Chem. Mater. 26, 5365-5373 (2014)

8. Kim, T. W. \& Choi, K.-S. Nanoporous $\mathrm{BiVO}_{4}$ photoanodes with dual-layer oxygen evolution catalysts for solar water splitting. Science 343, 990-994 (2014)

9. McDowell, M. T. et al. Improved stability of polycrystalline bismuth vanadate photoanodes by use of dual-layer thin $\mathrm{TiO}_{2} / \mathrm{Ni}$ coatings. J. Phys. Chem. $\mathrm{C} 118$, 19618-19624 (2014).

10. Lichterman, M. F. et al. Enhanced stability and activity for water oxidation in alkaline media with bismuth vanadate photoelectrodes modified with a cobalt oxide catalytic layer produced by atomic layer deposition. J. Phys. Chem. Lett. 4, 4188-4191 (2013). 
11. Ding, C. et al. Visible light driven overall water splitting using cocatalyst/BiVO photoanode with minimized bias. Phys. Chem. Chem. Phys. 15, 4589-4595 (2013).

12. Abdi, F. F., Firet, N., Dabirian, A. \& van de Krol, R. Spray-deposited Co-Pi Catalyzed $\mathrm{BiVO}_{4}$ : a low-cost route towards highly efficient photoanodes. Mater. Res. Soc. Symp. Proc. 1146, 7-12 (2012).

13. Zhai, P. et al. Net primary energy balance of a solar-driven photoelectrochemical water-splitting device. Energy Environ. Sci. 6, 2380-2389 (2013).

14. Modestino, M. A. et al. Robust production of purified $\mathrm{H}_{2}$ in a stable, self-regulating, and continuously operating solar fuel generator. Energy Environ. Sci. 7, 297-301 (2014).

15. Abdi, F. F. et al. Efficient solar water splitting by enhanced charge separation in a bismuth vanadate-silicon tandem photoelectrode. Nat. Commun. 4, 2195 (2013).

16. Seabold, J. A. \& Choi, K.-S. Efficient and stable photo-oxidation of water by a bismuth vanadate photoanode coupled with an iron oxyhydroxide oxygen evolution catalyst. J. Am. Chem. Soc. 134, 2186-2192 (2012).

17. Chen, L. et al. Mo-doped $\mathrm{BiVO}_{4}$ photoanodes synthesized by reactive sputtering. ChemSusChem. 8, 1066-1071 (2015).

18. Haussener, S. et al. Modeling, simulation, and design criteria for photoelectrochemical water-splitting systems. Energy Environ. Sci. 5, 9922-9935 (2012).

19. Abdi, F. F., Firet, N. \& van de Krol, R. Efficient $\mathrm{BiVO}_{4}$ thin film photoanodes modified with cobalt phosphate catalyst and W-doping. ChemCatChem. 5, 490-496 (2013)

20. Smith, W. A., Sharp, I. D., Strandwitz, N. C. \& Bisquert, J. Interfacial band-edge energetics for solar fuels production. Energy Environ. Sci. 8, 2851-2862 (2015).

21. Hardee, K. L. \& Bard, A. J. Semiconductor electrodes: X. Photoelectrochemical behavior of several polycrystalline metal oxide electrodes in aqueous solutions. J. Electrochem. Soc. 124, 215-224 (1977).

22. Myung, N. et al. Tailoring interfaces for electrochemical synthesis of semiconductor films: $\mathrm{BiVO}_{4}, \mathrm{Bi}_{2} \mathrm{O}_{3}$, or composites. J. Phys. Chem. C 115, 7793-7800 (2011).

23. Warren, S. C. et al. Identifying champion nanostructures for solar water-splitting. Nat. Mater. 12, 842-849 (2013).

24. Howell, S. L. et al. Spatial mapping of efficiency of GaN/InGaN nanowire array solar cells using scanning photocurrent microscopy. Nano Lett. 13, 5123-5128 (2013).

25. Economou, N. J., Mubeen, S., Buratto, S. K. \& McFarland, E. W. Investigation of arrays of photosynthetically active heterostructures using conductive probe atomic force microscopy. Nano Lett. 14, 3328-3334 (2014).

26. Jain, A. et al. Commentary: the materials project: a materials genome approach to accelerating materials innovation. APL Mater. 1, 011002 (2013).

27. Persson, K. A., Waldwick, B., Lazic, P. \& Ceder, G. Prediction of solid-aqueous equilibria: scheme to combine first-principles calculations of solids with experimental aqueous states. Phys. Rev. B 85, 235438 (2012)

28. Jeon, T. H., Choi, W. \& Park, H. Cobalt-phosphate complexes catalyze the photoelectrochemical water oxidation of $\mathrm{BiVO}_{4}$ electrodes. Phys. Chem. Chem. Phys. 13, 21392-21401 (2011).

29. Park, Y., Kang, D. \& Choi, K.-S. Marked enhancement in electron-hole separation achieved in the low bias region using electrochemically prepared Mo-doped $\mathrm{BiVO}_{4}$ photoanodes. Phys. Chem. Chem. Phys. 16, 1238-1246 (2014).

30. Zhong, D. K., Choi, S. \& Gamelin, D. R. Near-complete suppression of surface recombination in solar photoelectrolysis by 'Co-Pi' catalyst-modified $\mathrm{W}: \mathrm{BiVO}_{4}$. J. Am. Chem. Soc. 133, 18370-18377 (2011).

31. Han, L. et al. Optimization of amorphous silicon double junction solar cells for an efficient photoelectrochemical water splitting device based on a bismuth vanadate photoanode. Phys. Chem. Chem. Phys. 16, 4220-4229 (2014).

32. Gerischer, H. Electrochemical behavior of semiconductors under illumination. J. Electrochem. Soc. 113, 1174-1182 (1966).

33. Park, H. S., Leonard, K. C. \& Bard, A. J. Surface interrogation scanning electrochemical microscopy (SI-SECM) of photoelectrochemistry at a $\mathrm{W} / \mathrm{Mo}-\mathrm{BiVO}_{4}$ semiconductor electrode: quantification of hydroxyl radicals during water oxidation. J. Phys. Chem. C 117, 12093-12102 (2013).

34. Kweon, K. E. \& Hwang, G. S. Structural phase-dependent hole localization and transport in bismuth vanadate. Phys. Rev. B 87, 205202 (2013).

35. Kweon, K. E. \& Hwang, G. S. Surface structure and hole localization in bismuth vanadate: a first principles study. Appl. Phys. Lett. 103, 131603 (2013).

36. Pourbaix, M. Atlas of electrochemical equilibria. in Aqueous Solutions (National Association of Corrosion Engineers, 1974).
37. Mazin, I. I., Liechtenstein, A. I. \& Ivanov, O. V. Breathing instability and disproportionation of $\mathrm{Bi}^{4+}$ ions in $\mathrm{BaBiO}_{3}$. Ferroelectrics 164, 169-175 (1995).

38. Hautier, G., Fischer, C., Ehrlacher, V., Jain, A. \& Ceder, G. Data mined ionic substitutions for the discovery of new compounds. Inorg. Chem. 50, 656-663 (2011).

39. Jain, A. et al. A high-throughput infrastructure for density functional theory calculations. Comp. Mater. Sci. 50, 2295-2310 (2011).

40. Bergerhoff, G. \& Brown, I. D. in Crystallographic Databases. (eds Allen, F. H et al.) (Hrsg) (International Union of Crystallography, 1987).

41. Buffle, J., Zhang, Z. \& Starchev, K. Metal flux and dynamic speciation at (bio)interfaces. Part I: critical evaluation and compilation of physicochemical parameters for complexes with simple ligands and fulvic/humic substances. Environ. Sci. Technol. 41, 7609-7620 (2007).

42. Kresse, G. \& Furthmuller, J. Efficiency of $a b$ initio total energy calculations for metals and semiconductors using a plane-wave basis set. Comp. Mater. Sci. 6 15-50 (1996).

43. Blochl, P. E. Projector augmented-wave method. Phys. Rev. B 50, 17953-17979 (1994).

44. Perdew, J. P., Burke, K. \& Ernzerhof, M. Generalized gradient approximation made simple. Phys. Rev. Lett. 77, 3865-3868 (1996).

45. Wang, L., Maxisch, T. \& Ceder, G. Oxidation energies of transition metal oxides within the GGA + U framework. Phys. Rev. B 73, 195107 (2006).

46. Persson, K. A., Waldwick, B., Lazic, P. \& Ceder, G. Prediction of solid-aqueous equilibria: scheme to combine first-principles calculations of solids with experimental aqueous states. Phys. Rev. B 85, 235438 (2012).

47. Ong, S. P., Wang, L., Kang, B. \& Ceder, G. Li-Fe-P-O $\mathrm{O}_{2}$ phase diagram from first principles calculations. Chem. Mater. 20, 1798-1807 (2008).

48. Sleight, A. W., Chen, H. Y., Ferretti, A. \& Cox, D. E. Crystal growth and structure of $\mathrm{BiVO}_{4}$. Mater. Res. Bull. 14, 1571-1581 (1979).

\section{Acknowledgements}

This study is based on work performed at the Joint Center for Artificial Photosynthesis, a DOE Energy Innovation Hub, supported through the Office of Science of the U.S. Department of Energy under Award Number DE-SC0004993. Imaging work at the Molecular Foundry was supported by the Office of Science, Office of Basic Energy Sciences, of the U.S. Department of Energy under contract number DE-AC02-05CH11231. The EC-AFM part of this work was supported in part by the Laboratory Directed Research and Development Program of Lawrence Berkeley Nationa Laboratory under U.S. Department of Energy contract number DE-AC02-05CH11231. V.K. and F.M.T. acknowledge support from the $\mathrm{BaCaTeC}$ programme, project number 2015-1. Craig Jones from Agilent Technologies, Inc. is greatly acknowledged for his help with ICP-MS measurements.

\section{Author contributions}

F.M.T., F.A.H. and I.D.S. conceived of and designed this study. F.M.T., J.K.C., V.K. M.T.M., D.L., C.A., J.W.B. and I.D.S. performed the experiments. F.M.T., J.K.C., N.J.B., K.M.Y., J. Yang, F.A.H. and I.D.S. analysed and interpreted the data. J. Yu and K.A.P. performed the theoretical calculations and associated analysis. L.C., M.R.S. and J.S. provided some of the materials used for preliminary testing. All authors contributed to the final version of the manuscript.

\section{Additional information}

Supplementary Information accompanies this paper at http://www.nature.com/ naturecommunications

Competing financial interests: The authors declare no competing financial interests

Reprints and permission information is available online at http://npg.nature.com/ reprintsandpermissions/

How to cite this article: Toma, F. M. et al. Mechanistic insights into chemical and photochemical transformations of bismuth vanadate photoanodes. Nat. Commun. 7:12012 doi: 10.1038/ncomms12012 (2016).

This work is licensed under a Creative Commons Attribution 4.0 International License. The images or other third party material in this article are included in the article's Creative Commons license, unless indicated otherwise in the credit line; if the material is not included under the Creative Commons license, users will need to obtain permission from the license holder to reproduce the material. To view a copy of this license, visit http://creativecommons.org/licenses/by/4.0/ 